\title{
El hispanismo norteamericano a través de la colección de objetos: William H. Prescott (1796-1859) y sus calaveras aztecas
}

\author{
( Nina Gerassi-Navarro \\ Tufts University, Estados Unidos \\ Nina.gerassi@tufts.edu
}

Recibido: 28/7/2020. Aceptado: 15/12/2020.

\begin{abstract}
Resumen
Este trabajo analiza la relación entre los objetos y la reconstrucción historiográfica en la Historia de la conquista de México (1843) del hispanista norteamericano William H. Prescott. Entre los objetos que componen su vasto archivo documental, se encuentran dos cráneos indígenas que encargó de México. Influenciado por el auge de la craneología a principios del siglo XIX, el interés en las calaveras tenía una doble función: sustentar su narrativa histórica sobre las culturas pre-hispánicas y contribuir a un proyecto científico más vasto. $\mathrm{Al}$ analizar cómo se entrecruzan los discursos científicos e historiográficos durante los inicios del hispanismo norteamericano, este artículo explora cómo un objeto ayuda a definir una cultura, y muestra cómo la historia de los objetos y sus transformaciones son inseparables del discurso que los nombra.
\end{abstract}

Palabras clave: Prescott; historia de México; hispanismo; craneología, la colección de objetos.

\section{North American Hispanism Through the Collection of Objects: William H. Prescott (1796-1859) and his Aztec Skulls}

\footnotetext{
Abstract

This article analyzes the relationship between objects and historiography in the History of the Conquest of Mexico (1843) by US Hispanist, William H. Prescott. Among the objects comprising his vast archival documentation were two indigenous skulls he requested from México. Influenced by the success of craniology during the early nineteenth century, Prescott's interest in the skulls was twofold: to help construct a historical narrative about pre-Hispanic cultures and to contribute to a larger scientific project. Focusing on the interweaving of the discourses of science and history during the early stages of US Hispanism, this article explores the ways in which an object helps
} 
define a culture and shows how the history of objects and their transformations are inseparable from the discourse that names them.

Keywords: Prescott; history of Mexico; Hispanism; craniology; the collection of objects.

En 1840, el reconocido hispanista bostoniano, William Hickling Prescott, quien estaba trabajando en su monumental Historia de la conquista de México, le escribió a su amigo Ángel Calderón de la Barca, Primer Ministro de España en México, para pedirle un favor. Quería saber si le podía conseguir un cráneo azteca:

I have a request somewhat singular to make. Dr. Warren, the most eminent surgeon in this part of the country, has a very large and complete collection of skulls of the various American races, arranged in the Medical College. One only is wanting, the ancient Mexican, although he has several Peruvian. I should be very glad if you could meet with an Aztec head to supply the link in this craniological chain, as it would not only be a service to science, but to myself, as I am desirous to compare the formation of it with that of the other races. If you ever meet with such, I will thank you to send it to me. You will think my letter as full of notions as a Yankee pedlar's pack. But I have taken you at your word, and ask freely anything that I want (Prescott en Wolcott, 1925: 137).

El modo en que Prescott formula su peculiar pedido es revelador: en primer lugar, lo presenta como un pedido para la ciencia -el cráneo tiene una función científica, la de completar la colección de razas americanas de John Collins Warren, primer anestesiólogo y fundador del museo anatómico de la Universidad de Harvard-. En varias cartas Prescott le repetirá a Calderón que su pedido era para la ciencia, "in the cause of science" (Prescott en Wolcott, 1925: 186). Pero el cráneo era también para Prescott, quien estaba "desirous" de compararlo con el de otras razas, aunque no explica qué tipo de comparación pretende hacer ni aclara si ha estudiado otros cráneos. También resulta llamativo el modo en que imagina conseguir dicho cráneo: "if you ever meet with such", como si el obtener una cabeza azteca fuera el resultado de un hallazgo casual e imprevisto que no implicara una búsqueda cuidadosa. De hecho, casi un año más tarde, en mayo de 1841, frustrado, el ministro Calderón le escribiría a Prescott informándole que aún no había podido conseguir el cráneo porque para ello debían desenterrar una tumba y el proceso de autorizaciones para hacerlo era bastante complicado. El comentario final de la cita termina prácticamente por anular el sentido original del encargo como un pedido serio y respetable, cuando Prescott lo califica casi como una ocurrencia. El cráneo en este sentido sería una pieza más no de una colección científica, sino de su museo de curiosidades, "a Yankee pedlar's pack".

La ambivalencia en el modo en que Prescott presenta su pedido-desde un objeto de ciencia a una mera curiosidad-abre un espacio que impide clasificar y definir el objeto en términos claros y precisos, aludiendo simultáneamente al entrecruzamiento de discursos que sucede en este período. Esta oscilación desnaturaliza el objeto, poniendo de manifiesto algunas de las transformaciones posibles que se le pueden adjudicar a un objeto cuando es desplazado de su contexto. ¿Debemos pensar en el cráneo como un objeto de estudio, que merece atención rigurosa o como una pieza más de una colección singular?

\footnotetext{
1 “Tengo un pedido algo peculiar para hacerle. El cirujano más eminente de esta parte del país, el Dr. Warren, tiene una gran y completa colección de cráneos de varias razas americanas, desplegada en la Escuela de Medicina. Una sola le falta, la antigua mexicana, aunque tiene varias peruanas. Me daría muchísimo gusto si pudiera encontrar una cabeza azteca para completer su cadena craneológica, ya que sería no solo un servicio para la ciencia sino también para mí, pues estoy deseoso de comparar su formación con la de otras razas. Pensará que mi carta está llena de rarezas como la de un vendedor ambulante. Pero le he tomado la palabra, y le pido con total libertad todo lo que se me ocurra". Todas las traducciones, a menos que se aclare, son de la autora.
} 
La esposa del ministro Calderón, Frances Calderón de la Barca (popularmente conocida como Fanny Calderón), una de las grandes cronistas de la vida mexicana durante ese período, percibió la oscilación que lo motivaba a Prescott y, cumplido el pedido, le escribe a su amigo con un tono juguetón: "I have at this moment two skulls beside me, not of Aztecs however but of Ottimiti Indians, who little thought that you were destined to disturb their repose" (Prescott en Wolcott, 1925: 137). ${ }^{2}$ Otomí no es lo mismo que azteca, y Fanny Calderón, después de estar viviendo más de un año en México lo sabía bien, pero intuyó correctamente que la exactitud científica le preocupaba menos a Prescott que el satisfacer su "deseo" de tener entre manos la cabeza de algún indígena mexicano. También vale notar que reformula el "deseo" de Prescott en términos de "destino", asociándolo con la retórica del "destino manifiesto" de los Estados Unidos (O'Sullivan, 1845).

¿Qué podía representar el cráneo para Prescott? ¿Qué tipo de información podía brindarle? ¿Y qué tipo de estudio comparativo pensaba hacer si no era antropólogo, arqueólogo ni científico? Los objetos sin duda son documentos materiales del pasado, pero no son indicadores transparentes; su función y significado necesitan ser decodificados. Ese proceso de ordenar, clasificar y describir un objeto, nos permite aprender sobre el objeto mismo, pero sobre todo nos enseña los valores y modos de pensar del que ejecuta la decodificación (Ulrich, 2015).

El estudio de los objetos se ha abordado desde una multiplicidad de perspectivas (filosófica, psicoanalítica, antropológica, económica, artística) según el énfasis del acercamiento, ya sea en la percepción del individuo, en el modo de circulación del objeto, su valor de cambio, su materialidad, su lugar en la memoria colectiva, etc. Dichos estudios subrayan el aspecto relacional del sujeto-objeto, que está enmarcado en un determinado contexto espacio-temporal. Para superar ese aspecto relacional que en cierta manera limita el acercamiento al objeto mismo, Arjun Appadurai propone seguir el recorrido de los objetos y sus transformaciones porque los "significados están inscritos en sus formas, usos y trayectorias" (Appadurai, 1991: 19), idea que Bruno Latour comparte (2000: 10). El promotor de la teoría de las cosas (Thing theory), Bill Brown (Brown, 2001), distingue entre objeto y cosa, señalando que el objeto se caracteriza por su funcionalidad. Cuando el objeto agota su función utilitaria, argumenta Brown, se desprende de su código social, de su definición, se vuelve cosa, lo cual permite que surjan nuevas formas de expresión, nuevas reflexiones sobre cómo los objetos inanimados determinan a los humanos.

Sobre la base de estas consideraciones este artículo se enfoca en el modo en que leemos una cultura a través de sus objetos; analiza de qué manera un objeto informa e inclusive puede ayudar a definir una cultura. Para ello me detengo en los intersticios y alteraciones que ocurren en el traslado de un objeto, ya que son los desplazamientos los que permiten la materialización de nuevos significados y analizo de qué forma el discurso científico colabora con las interpretaciones asignadas. ¿Cómo adquiere entonces un objeto-en este caso dos calaveras ${ }^{3}$ a partir del viaje, un valor inmanente que Prescott traslada al discurso historiográfico? ¿Cuáles son los criterios empleados en la lectura de los objetos que supuestamente permiten acceder a un conocimiento más profundo de una cultura, y que, en el caso de Prescott, le permitieron imaginar y definir la cultura hispanoamericana?

2 "Tengo en este momento a mi lado dos cráneos, no de aztecas sino de indios otomí, quienes nunca sospecharon que estabas destinado a interrumpir su reposo".

3 Si bien "calavera” y “cráneo" se usan indistintamente (en inglés skull para ambos), "cráneo” es usado en el campo científico y "calavera” es usado de forma más general. En la ciencia, el esqueleto de la cabeza comprende dos partes: el cráneo o caja ósea (que protege el encéfalo) y la cara. Véase Henri Rouvière y André Delmas (2006: 39). Agradezco a Julia Rubione por la referencia. 


\section{Los contextos}

Durante el siglo XIX, impulsados por el trabajo del geógrafo y naturalista alemán Alexander von Humboldt, viajeros, científicos y arqueólogos desfilaron por el continente americano con el fin de estudiar su naturaleza y culturas pre-hispánicas. Humboldt, quien había recorrido el continente durante cinco años (1799-1804), regresó a Europa con cuarenta y dos cajas colmadas de plantas, insectos, animales, semillas, rocas, minerales, artefactos indígenas, cerámicas, telas y mucho más, a partir de lo cual elaboró un complejo sistema de conexiones culturales y científicas que daría origen a una nueva cosmografía y "conciencia universal" (Ette, 2019: 2). El desarrollo de nuevas disciplinas científicas, junto con la creación de museos y sociedades científicas que deseaban conocer esas culturas rápidamente, promovió la colección etnográfica. Desde artefactos culturales hasta partes del cuerpo, los objetos se convirtieron en una forma de documentación, prueba, que anclaba el análisis de esas culturas no-europeas y facilitaba su clasificación y catalogación.

El cráneo humano fue uno de los objetos más buscados para el estudio de las razas humanas. Su estructura ósea resistía la descomposición, lo cual permitía un análisis más exacto de su forma, era fácil de trasladar y, al proteger el cerebro, se le asignaba una conexión con las facultades mentales (Franey, 2001). En Estados Unidos, Samuel George Morton fue uno de los primeros en dedicarse al estudio detallado de cráneos. Como fundador de la escuela norteamericana de etnografía, Morton empleó un método racional para la medición de cráneos. Su innovación consistió en medir la cavidad interior del cráneo en lugar de enfocarse en su perfil, ángulos y base. Para ello, elaboró un sistema de medición con mercurio líquido y semillas de pimienta. Morton examinó cientos de cráneos, sobre todo indígenas con el fin de diferenciar las razas humanas. Su colección llegó a ser una de las más grandes del mundo, superando los novecientos cráneos. Según el naturalista, cuanto mayor era la cavidad craneal mayor el cerebro, y como el cerebro reflejaba la capacidad mental de la persona, su conclusión fue que la raza caucásica se caracterizaba por "la facilidad con la que alcanza su máximo potencial intelectual" (Morton, 1839: 5). Morton denominó su trabajo craneometría, una rama de la antropometría que se concentraba en el estudio y medición volumétrica de los cráneos. Ann Fabian analiza el trabajo de Morton ilustrando el modo en que sus conclusiones científicas se mezclaron con prejuicios de la época, inexactitudes y subjetividades que dieron origen al racismo científico (Fabian, 2010). Por ello los historiadores de la cultura material enfatizan que lo que importa no son los objetos en sí, sino la interpretación que se les asigna y las relaciones que se establecen a partir de ellos como análisis cultural. La importancia asignada al cráneo humano en esta época quizás explique el deseo de Prescott: su valor es evidencia del pasado que se propone describir, un documento valioso para su reconstrucción e interpretación del pasado pre-hispánico.

El interés científico e histórico por las culturas pre-hispánicas en Estados Unidos coincidió además con el desarrollo de una narrativa nacional de consolidación política. Siguiendo el mandato de su destino manifiesto, Estados Unidos ensanchaba sus fronteras geográficas. La compra de Luisiana (1803) había duplicado su extensión territorial que continuaría hacia el Pacífico y el sur. El vínculo con Hispanoamérica no solo fortalecía su separación de Europa sino que también permitía que se articulara el discurso de "nación de futuridad" (O'Sullivan, 1839: 429). ${ }^{4}$ Los norteamericanos se

4 O'Sullivan estaba convencido de que los Estados Unidos tenían un mandato divino de diseminar la democracia republicana por todo el continente, sobre todo en el norte, porque representaban "la nación del progreso, de libertades individuales, de emancipación universal" ("the nation of progress, of individual freedom, of universal enfranchisement"). Esta es la base de argumentación para su ensayo posterior de 1845 acerca de la anexión de Texas en que usó el término “destino manifiesto" para definir el mandato de los Estados Unidos. 
veían como el nuevo modelo de nación, republicana y democrática, con una política de excepcionalismo ratificada por la Providencia que solo miraba hacia el futuro, como lo reafirmó el periodista John O'Sullivan:

Our national birth was the beginning of a new history, the formation of progress of an untried political system, which separates us from the past and connects us with the future only [...] we confidently assume that our country is destined to be the great nation of futurity (O'Sullivan, 1839: 426).

El problema era que las naciones hispanoamericanas estaban lejos del modelo anglosajón: tenían un pasado mucho más complejo, y sus valores culturales y mezcla racial en nada coincidían con el imaginario norteamericano. Basta recordar el violento traslado que el gobierno federal les impuso a más de cien mil indígenas, colocándolos en reservas más allá del río Mississippi para evitar que se mezclaran con el resto de la población. ${ }^{6}$ Estudiar entonces el pasado de España, el gran imperio que había perdido control de sus colonias, era una manera de acercarse al mundo hispánico y reafirmar el proyecto nacional de los Estados Unidos. Los norteamericanos admiraban profundamente la historia y el esplendor cultural de España. Por ello, su enfoque se concentró en la reconstrucción de ciertos momentos de su pasado como la expulsión de los árabes, la llegada a América, la conquista de México, el descubrimiento del imperio inca, eventos que en su conjunto impulsaron a España a ser una gran potencia. A la vez, la exploración de esos hechos les permitió formular una explicación de su eventual caída y proyectar los ideales emblemáticos de su país. España interesaba porque era esencialmente la antítesis de los Estados Unidos (Kagan, 1996; Jaksic, 2007).

Tanto en Estados Unidos como en Hispanoamérica, el discurso hispanista surge con el proceso de consolidación nacional. Sin embargo, el acercamiento al estudio de las lenguas, literaturas y culturas hispánicas no se extiende uniformemente por el continente. Su desarrollo multifocal junto con el contexto político, socio-histórico y cultural de cada lugar genera un movimiento complejo, lleno de tensiones (Zuleta, 1992). En Latinoamérica los debates acerca del proyecto nacional se funden con el análisis y valoración de la cultura española. Para algunos autores, como Domingo F. Sarmiento, Carlos María de Bustamante y José María Samper significó un cuestionamiento y rechazo de lo español, mientras que, para otros, como Andrés Bello, José María Vergara y Lucas Alamán implicó reivindicar la herencia española (Jaramillo, 1986). La valoración de lo español en Hispanoamérica tampoco permanece inalterable, ya que a medida que evolucionan los debates ideológicos sobre lo nacional también se transforman los ejes definitorios del hispanismo. ${ }^{7}$ Para España, el hispanismo fue un modo generalizado de afianzar su conexión con las Américas y recuperar la hegemonía de su poder simbólico a través de la lengua, mientras que para Estados Unidos representó un instrumento de poder discursivo que le permitió articular su excepcionalismo. ${ }^{8}$ En este contexto, la reconstrucción norteamericana de la historia antigua de Hispanoamérica tuvo un significado determinante en el modo

5 "Nuestro origen nacional fue el principio de una nueva historia, la creación del progreso de un sistema político aún no probado, que nos separa del pasado y nos conecta únicamente al futuro [...] con seguridad asumimos que nuestro país está destinado a ser la gran nación de futuridad".

6 Dicho traslado se conoce como el tratado de Remoción indígena o Indian Removal Act (1830). Y apenas dos años antes de que Prescott enviara su carta a Calderón, entre 1838 y 1839 , el estado de Georgia desplazó a los cherokees que se habían resistido en una brutal travesía llamada el sendero de lágrimas o Trail of Tears, que acabó con $25 \%$ de la población indígena (Filler, 1988).

7 Sobre la evolución y desarrollo del hispanismo a lo largo de los siglos, véase Moraña (2005) y Cortés y Gómez (2015).

8 La ideología del excepcionalismo americano surge a partir de la revolución americana en la que los Estados Unidos declaran ser la primera nueva nación basada en libertad, igualdad, individualidad. Véase Seymour Martin Lipset (1996). 
de entender la cultura hispana y definir las relaciones futuras con las antiguas colonias españolas. Ese fue justamente el enfoque de Prescott al emprender su Historia de la conquista de México.

\section{Una mirada propia}

Con la publicación de su Historia del reinado de los reyes católicos Fernando e Isabel (1837), Prescott se había convertido en uno de los hispanistas más importantes de los Estados Unidos, junto con Washington Irving, Henry Wadsworth Longfellow y George Ticknor, entre otros. Esta elite cultural bostoniana de brahmanes (a excepción de Irving) sostenía fuertes vínculos con la Universidad de Harvard. Formaban además parte de una enérgica red intelectual en la que se entrecruzaban figuras literarias, políticos y científicos que determinó el discurso intelectual norteamericano. A través de la fundación de revistas como The North American Review y la creación de sociedades formales e informales para el avance de la cultura y las ciencias, esta elite afianzó la circulación de un conocimiento acerca del mundo y de la cultura norteamericana que influenciaría al país por décadas.?

Prescott fue uno de los primeros hispanistas en desplazar su mirada de España a Latinoamérica. Intuyó que Latinoamérica sería de mayor relevancia para los Estados Unidos, ya que el vínculo con España era en parte lo que moldearía el futuro latinoamericano (Ticknor; 1865: 220). Primero con su Historia de la conquista de México (1843) y luego con su Historia de la conquista de Perú (1847), ilustró vívidamente la grandeza y las fallas del imperio español que llevarían a su eventual caída. Este juego de sutilezas en las que yuxtapone la decadencia española con el progreso norteamericano es lo que Kagan llama "el paradigma de Prescott" (Kagan, 325). Dicho paradigma estaba sólidamente anclado en una vasta colección de documentos históricos, pero narrado a través de un discurso ameno y entretenido.

Influenciado por el impulso científico, Prescott construye su retrato del pasado prehispánico a partir de un impresionante archivo, logrado a base de la red de contactos internacionales que había establecido durante su investigación sobre los reyes católicos. De esta manera, sin nunca abandonar los Estados Unidos y luchando con una ceguera progresiva, hace que la documentación venga hacia él. Recibe cajas y cajas de manuscritos, libros, informes, documentos, cartas, facsímiles, ilustraciones, grabados y mapas; todo lo pertinente para armar su reconstrucción de la conquista. Su red de colaboradores se extiende por toda Europa: Pascual de Gayangos; Martín Fernández de Navarrete; Arthur Middleton, los historiadores Friedrich Wilhelm Lembke y Henri Ternaux Compans, y el conde Camaldoli de Nápoles entre muchos otros. ${ }^{10}$ Prescott es un ávido lector que posee un "apetito insaciable" de material (Eipper, 2000: 421). De México, sin embargo, solicitó comparativamente poco, ya que basó su reconstrucción principalmente en las autoridades coloniales y españolas (Cockcroft, 1968). Primero contrata la firma inglesa Manning \& Marshall. ${ }^{11}$ Sin embargo, tan solo después de un mes la firma le informó que era imposible conseguir material en México porque

9 The North American Review fue la primera revista literaria de los Estados Unidos, fundada en Boston en 1815. Allí publicaron las figuras literarias de mayor relevancia junto con políticos como John Adams, George Bancroft y Daniel Webster. Además de las instituciones nacionales de investigación, como la American Association for the Advancement of Science (1848) y la American Philosophical Society, fundada en 1743 por Benjamin Franklin, entre cuyos miembros figuraban Charles Darwin, Louis Pasteur, Elizabeth Cary Agassiz, entre otros, había varias asociaciones informales, siendo la más conocida el renombrado Club de los Sábados fundada por Ralph Waldo Emerson en 1855.

10 Para una detallada lista de sus colaboradores véase Peter O. Koch (2016) y sobre la recepción de su trabajo en México, Leila Gómez (2015).

11 Robert Manning y William Marshall, ciudadanos ingleses residentes en México, fueron instrumentales en desarrollar el poder británico en México. Su firma se encargó de negociar prestamos, financiación, además de la importación y exportación de bienes entre compañías inglesas y las elites mexicanas (Tenenbaum, 1979). 
había demasiada confusión y, como ya se lo había anticipado Ángel Calderón, todo en México era puro desorden y no se podía confiar en los mexicanos, quienes siempre respondían con el eterno refrán "mañana, mañana", que en realidad significaba en el próximo siglo o nunca (Calderón en Wolcott, 1925: 112).

Ante las dificultades para obtener documentos en México, Prescott recurrió a sus amistades locales, miembros de la elite intelectual: los Cortina, Lucas Alamán, Manuel Goroztiza y sobre todo, a sus queridos amigos diplomáticos, Ángel y Fanny Calderón. Aparte de algún manuscrito inédito, les pidió otro tipo de material: ilustraciones, cuadros, grabados, mapas, descripciones de paisajes, dibujos, fotos (para lo cual le regaló un daguerrotipo a Ángel Calderón), inclusive un fragmento del sudario de Cortés. Estos objetos son en su mayoría materiales visuales que lo ayudan a ver, imaginar la realidad del pasado y anclar su entramado de las relaciones culturales, sociales y económicas en un marco accesible para sus lectores.

Curiosamente, pide muy pocos objetos pre-hispánicos. No le interesan las cerámicas, decoraciones, telas o herramientas de la cultura mexica; solo insiste en la calavera azteca. ${ }^{12}$ En la misma carta en la que pide los cráneos, le recuerda a Calderón sus otros pedidos: un mapa, un recorte con el autógrafo de Cortés, pregunta por la autenticidad del grabado recibido de Moctezuma y regatea el precio de un retrato de Cortés. El hecho de que Prescott se compare con un vendedor ambulante (a Yankee Pedlar's pack) en la cita inicial de este ensayo es significativo. Además de vender todo tipo de objetos, a principios de siglo el yankee pedlar, personaje típico de Nueva Inglaterra, se había convertido en una figura de relevancia cultural que traía novedades e informaba sobre los acontecimientos políticos y sociales; era un especie de encargado de diseminar la civilización y cultura por el interior del país a través de sus objetos (Dolan, 1964). ${ }^{13}$ Prescott también instruye a la población norteamericana sobre México y en este sentido, al igual que para el vendedor ambulante, su variada colección de documentos y objetos servía para fijar su narración.

En su estudio sobre la cultura material, Richard Grassby explica que los objetos nos ayudan a comprender cómo se veían los individuos de una cultura a sí mismos, sus valores y calidad de vida (594). Si bien Prescott quiere hacer un retrato completo de la cultura mexica, no le interesa ahondar en un estudio antropológico ni entender cómo se veían los mexicas a sí mismos. Su retrato sirve para contrastar su cultura con la civilización y progreso de los españoles. A Prescott le interesan los objetos que refuercen su narrativa. Como historiador, los objetos que le sirven son sobre todo los documentos escritos, recordándonos que el estudio de los objetos está determinado por la disciplina que la contiene (Hicks y Beaudry, 2010: 4).

La Historia de la conquista de México tuvo un éxito inmediato y se convirtió en un referente clave para la cultura anglosajona. Comenzando con la fundación del imperio azteca hasta la llegada de los españoles a México y su conquista, Prescott consolida una visión romántica de la historia épica a través de una combinación de elementos: "a daring, chivalrous enterprise, stupendous achievements worthy of an age of knighterrantry, a magical country, the splendors of a rich barbaric court and extraordinary personal qualities in the hero" (Prescott, 1961 v. 2: 29). ${ }^{14}$ Influenciado por sus lec-

12 Mexica es el término usado por los mismos pueblos migratorios nahuas, quienes provienen de Aztlán y habitaron el valle del Anáhuac donde fundaron su capital México-Tenochtitlán, en el centro del Lago Texcoco. Hernán Cortés se refiere a los mexicas. Pero Alexander von Humboldt prefirió usar el término azteca, y desde entonces prevaleció entre los autores europeos el uso del término azteca para referirse a todos los habitantes del valle del Anáhuac.

13 El legado de los yankee pedlars se puede ver aún hoy en la multitud de tiendas y hoteles en Nueva Inglaterra con su nombre, además de festivales anuales en su honor. Para un análisis sobre su rol en la cultura de consumo de Nueva Inglaterra, véase Jaffee.

14 "una valiente empresa, logros estupendos dignos de la edad de los caballeros errantes, un país mágico, esplendores de una corte rica y bárbara con un héroe de cualdidades personales extraordinarias". 
turas de Walter Scott y René de Chateaubriand, Prescott vuelve la historia accesible y entretenida para el público general norteamericano. ${ }^{15}$ Los lectores siguen paso a paso las acciones del celebrado héroe Hernán Cortés en su brillante conquista. Pero son sus disquisiciones intercaladas las que dan sentido a su retrato y que establecen la distinción entre el triunfo español y el futuro de los Estados Unidos. Por ejemplo, poco después de su llegada a México, Cortés ofrece una misa frente a los curiosos nativos. Prescott comenta,

The Roman Catholic communion has, it must be admitted, some decided advantages over the Protestant, for the purpose of proselytism. The dazzling pomp of its service and its touching appeal to the sensibilities affect the imagination of the rude child of nature much more powerfully than the abstractions of Protestantism, which, addressed to the reason, demand a degree of refinement and mental culture in the audience to comprehend them (Prescott, 1961 V.2: 29).

El contraste es evidente: el despliegue del espectáculo frente a lo medido y sobrio; lo sensible frente a lo intelectual y razonado; estas son las cualidades que distinguen el catolicismo del protestantismo. Prescott reitera este tipo de comentario a lo largo de su historia una y otra vez, asegurando que la lectura del pasado sirva para ilustrar el presente y destacar el modelo de nación que ofrecía Estados Unidos. Los valores protestantes son los que eventualmente asegurarán el progreso porque confían en la razón del individuo. El católico se deja deslumbrar por el espectáculo, como un niño inocente e inculto, su comprensión en nada se relaciona con la razón que, según Prescott, es lo que distingue a ambas religiones. Evocando la futuridad del destino manifiesto y la empresa mesiánica de los Estados Unidos, Prescott entrelaza los discursos políticos y religiosos norteamericanos en su lectura del pasado mexicano.

Uno de los rasgos que la crítica más destacó de la obra de Prescott fue su capacidad para visualizar la historia. Humboldt, quien pasó un año en México, lo felicitó por esta cualidad, "You paint with success because you have seen with the eyes of the spirit and of the inner sense" (Humboldt en Peck, 1926: 81). ${ }^{17}$ Si Prescott -quien escribió su historia con la ayuda de un nictógrafo para compensar su ceguera parcial- logró pintar con vívidos colores el mundo donde se habían desarrollado las acciones que documenta, fue gracias a la enorme colección de documentos que reunió y a la ayuda de su amiga, Fanny Calderón. A pesar de su énfasis en las fuentes originales, Prescott jamás visitó México. Su evocación colorida la realiza Fanny. Ella le describe con lujo de detalles el paisaje, los edificios, las costumbres y vestimenta de la gente; sus ojos son los de Prescott:

If you please on the scenery, and let me know if you can without trouble a few of the indigenous, staple trees and shrubs of the grand tableland growth and of the tierra caliente, and their English names, if they have any. Could you not specify a bird or two, with their effect on the eye? You see I want to dip my pencil in your colours - the colours of truth, gently touched with fancy, at least feeling (Prescott en Wolcott, 1925: 240).

15 El proyecto intelectual de Prescott se asemeja al de Scott, en la medida en que su reconstrucción del pasado surge a partir de los valores impulsados por la revolución francesa, aunque Prescott los combinará con el excepcionalismo norteamericano. Sobre Scott véase Lukács.

16 “Hay que admitir que en función del proselitismo la comunión católica romana tiene algunas ventajas por encima de la protestante. La deslumbrante pompa de su servicio y su conmovedor atractivo a las emociones afectan la imaginación del rudo niño sin educación mucho mas enérgicamente que las abstracciones del protestantismo, que, dirigidas a la razón, demandan un grado de refinamiento y cultura mental de su público para que las comprendan". 17 "Usted pinta con éxito porque ha visto con los ojos del espíritu y con profunda comprensión".

18 "Por favor un poco sobre la escenografía y déjame saber, si no es muy inconveniente, cuáles son algunos de los principales árboles y arbustos indígenas de la gran meseta de la tierra caliente y sus nombres en inglés, si es que lo tienen. ¿Podrías quizás especificar uno o dos pájaros, y su efecto en la vista? Como verás quiero sumergir mi lapiz 
El tono juguetón e imagen fálica de la cita no disimulan que Prescott apela a la complicidad y ayuda de Fanny, quien publicará su colección de cartas sobre México el mismo año que Prescott con un prólogo suyo. ${ }^{19}$ Por su lúcido análisis histórico y cultural ambos libros serían también material de lectura obligatoria para los soldados norteamericanos unos años después al marchar a la guerra contra México (Johannsen, 1985: 148-150). Prescott depende de la mirada de su amiga para reconstruir visualmente la escenografía de su historia. Para recrear la barbarie de los aztecas recurre a las calaveras.

\section{El giro científico de la barbarie}

Teniendo en cuenta el estudio de las razas y culturas pre-hispánicas, el pedido de Prescott tiene un fin científico que además está cargado de simbología relacionada con la muerte, la finitud de la vida y el horror, poniendo de manifiesto que los artefactos tienen un valor práctico y simbólico, que incide en su valor jerárquico variable (Grassby: 594). Para las culturas europeas la máxima expresión de barbarie de las culturas mesoamericanas yacía en sus sangrientos rituales religiosos que incluían sacrificios humanos y la exhibición de calaveras. Prescott constantemente subraya la barbarie mexica aun cuando elogia su sofisticación cultural y sus impresionantes avances. Benjamin Keen clasifica la Historia de la conquista como una amalgama de actitudes pro y anti-indígenas (1990: 354). En sus notas personales Prescott confiesa que las antigüedades mexicanas son un tema detestable que tratará con mucha cautela (Prescott, 1839: 15). Describe su sistema de escritura y agricultura, sus avances matemáticos, su orfebrería, arquitectura, escultura, arte, es decir, todos los aspectos deslumbrantes de su cultura, sin dejar de intercalar adjetivación o referencias que refuercen su barbarie. Al hablar del complejo sistema político mexica declara que es más refinado "de lo que se podría haber esperado de una nación bárbara" (2001: 28); la solemne recepción que le ofrece Moctezuma a Cortés es "inesperada de un bárbaro" (2001: 400); al describir su maravilloso trabajo en alabastro cita a Torquemada, quien declara que la ornamentación de sus deidades era "el espantoso reflejo de sus almas" (2001: 107); y aun cuando no tiene ninguna crítica se refiere a ellos como "los feroces aztecas" (2001: 113). Una y otra vez resalta su barbarie aun cuando se trata de acciones que nada tienen de bárbaro.

Prescott estructura su mayor crítica de los mexicas cuando analiza su religión, eje de su organización política y social. Firme creyente en el poder del progreso que regía el pensamiento nacionalista norteamericano, sostiene que los aztecas estaban destinados a ser conquistados por sus "inherentes culpas morales" (Levin, 1959: 164). Esa culpa era la barbarie de su religión. Prescott presenta la religión azteca como una mezcla incongruente que emana de un pueblo culto que a la vez exhibía una "ferocidad indómita"(2001: 65). Su religión debe entenderse como mitología, "el esfuerzo del hombre inculto por explicar los misterios de su existencia" (2001: 48). Como mito, es una forma de pensamiento anterior a la civilización en la que narraciones de dioses y héroes justifican el origen de una cultura o ciertos hechos. Es un tipo de pensamiento que se opone al pensamiento científico y racional. Si bien reconoce que los aztecas no fueron caníbales, astutamente vincula sus sacrificios humanos al canibalismo para descalificarlos como civilización.

[T] hough, in truth, the Mexicans were not cannibals, in the coarsest acceptation of the term. They did not feed on human flesh merely to gratify a brutish appetite, but

en tus colores-los colores de verdad, ligeramente embellecidos por la fantasía, por lo menos con emoción". Sobre la relación escritural entre Prescott y Fanny Calderón véase Nina Gerassi-Navarro (2017).

19 De hecho, fue Prescott quien animó a Fanny a publicar su volumen titulado Life in Mexico y contactó a Charles Dickens para asegurar su publicación simultánea en Inglaterra. 
in obedience to their religion [...] Still, cannibalism, under any form, or whatever sanction, cannot but have a fatal influence on the nation, addicted to it. It suggests ideas so loathsome, so degrading to man, to his spiritual and immortal nature, that it is impossible the people who practise it should make any great progress in moral or intellectual culture. The Mexicans furnish no exception to this remark (Prescott, 2001: 68).

Esta incapacidad moral es la marca del salvaje. Prescott no se detiene en explicar el sentido o función de los sacrificios humanos, simplemente constata que la religión mexica los exigía. ${ }^{21}$ En este contexto, el cráneo azteca se convierte en la visualización material de su barbarie por ser un testimonio de las víctimas sacrificadas, desplegadas en los tzompantlis. ${ }^{22}$ Prescott lee esta diferencia entre el primitivismo azteca y el progreso de la civilización occidental en la materialidad de los cráneos.

El estudio científico de los cráneos se superpuso con la frenología. El neuroanatomista alemán Joseph Gall (1758-1828) sostuvo que las protuberancias del cráneo correspondían a determinados rasgos de personalidad. Su desarrollo del mapa frenológico permitía localizar las facultades mentales que correspondían a designadas zonas del cráneo, como si las características de la personalidad estuvieran grabadas en el cráneo. La frenología cayó en decadencia a mediados de siglo, pero en su momento ofreció corroboración de que por el tamaño y forma de su cerebro, la raza caucásica era superior a la amerindia.

Influenciado por estas teorías dominantes, el deseo de Prescott por obtener un cráneo indígena se puede entender como la posibilidad de corroborar la inferioridad de los aztecas. El tener entre manos un cráneo podría equipararse al análisis de un documento original. Los mapas frenológicos hacían palpable lo intangible. A menudo en las fotos de Gall y del mismo Warren, destinatario de los cráneos de Prescott, se los ve con una mano posada sobre el cráneo, como si el tacto permitiera una lectura más penetrante. Llama la atención el interés por tocar, palpar los cráneos, como si ese inner sense (profunda comprensión de la interioridad) que Humboldt tanto le elogió a Prescott se pudiera alcanzar a través del tacto; al igual que Hamlet, quien al tomar en sus manos la calavera del bufón Yorick revive su infancia. Por su parte, Ángel Calderón se queja de que el político mexicano Lucas Alamán, quien se había autodesignado protector de la tumba de Cortés y había escondido sus huesos por temor al vandalismo político, no le había dejado tocar el cráneo de Cortés cuando le mostró sus huesos (Calderón en Wolcott, 1925: 126). Esta necesidad de poseer, sentir el cráneo permitió asignarle un rasgo inmanente que solo se percibía a través del tacto que, a la vez, aumentó la fetichización de los cráneos durante esta época (Franey, 2001: 223).

En la carta que Prescott le envió a Warren junto con los cráneos, queda en evidencia que este no le había hecho ningún pedido, ni siquiera había expresado un deseo de añadir un cráneo azteca a su colección. Warren fundó el Museo Anatómico a partir de su colección privada de 160 cráneos. ${ }^{23}$ Prescott visitó dicha colección con su padre,

20 "Aunque en verdad los mexicanos no fueron caníbales en el sentido mas estricto de la palabra. No se alimentaron de la carne humana para gratificar un apetito salvaje, sino en obediencia a su religión [...] Sin embargo, el canibalismo, de cualquier tipo o bajo cualquier sanción, no puede tener más que un efecto fatal para la nación adicta a esta práctica. Sugiere ideas tan repugnantes, degradantes al hombre, a su naturaleza espiritual e inmortal, que es imposible que la gente que lo practicara pudiera lograr algun progreso en la cultura moral o intelectual. Los mexicanos no proporcionan ninguna excepción a esta declaración".

21 Los sacrificios tenían diferentes funciones, como asegurar la reconstrucción de un nuevo orden, representar un mito o acontecimiento histórico, o reforzar los pactos con sus dioses; estaban, además, íntimamente vinculados con las formas de producción y reproducción de la sociedad (ciclos agrícolas, medios de trabajo o guerras y conquistas), véase Zaid Lagunas Rodríguez (2004).

22 Los tzompantli eran altares de madera colmados de calaveras sangrientas. Vale notar que estos muros se asemejan a los que se encuentran en algunas iglesias o en las catacumbas de París, que no se leen como barbarie.

23 Además de fundar una de las revistas más importantes de medicina aún hoy, The New England Journal of Medicine, 
y mucho antes de que fuera donada, en 1841 , se asignó la tarea de completarla. Al enviarle su regalo a Warren le informó que él había notado que le faltaba un cráneo mexicano. Al no poder conseguir un cráneo azteca "genuino" le obsequiaba uno otomí, "una de las numerosas tribus salvajes" que habitaba el área central de México antes de la llegada de los mexicas. ${ }^{24}$ En la caja había dos cráneos, uno de mujer y otro de hombre. Uno, aclara Prescott, había sido extraído de la caja, pero el otro no había sido tocado. Su comentario alude al fetichismo creado alrededor del objeto, en este caso a la idea de pureza del cráneo que no ha sido estorbado, más allá de ser desenterrado y enviado a los Estados Unidos (Prescott en Warren, 24 de julio, 1841). Warren no prestó atención a la explicación de Prescott, ni comentó sobre su origen, ni transmitió el entusiasmo que podría suponer completar una colección. Tan solo afirmó en su diario, "Received 2 Mexican, Aztec skulls from W.H. Prescott esq. who, without a request took the trouble to send for them from the valley of Mexico" (Warren, 24 de julio, 1841). ${ }^{25}$ Tampoco hace caso de la aclaración de Prescott acerca del origen de los cráneos. El envío surge de un deseo o "antojo" de Prescott, que él justifica como ayuda a Warren. Conseguir un cráneo era para Prescott su modo de contribuir a la colección científica, cuya interpretación él ya tenía entre manos en su Historia.

Al reflexionar sobre los museos, Theodor Adorno (1961: 187) observa que para exponer un objeto primero es preciso eliminar el contexto en el que se origina la colección, lo cual lleva a la "neutralización de la cultura." Toda colección desplaza el objeto de su lugar de origen y esta transición le permite adquirir otro significado. La colección nunca constituye un todo, sino que responde a intereses tan variados como los distintos individuos o instituciones que se proponen realizarla (Torres Monroy, 2000: 32). El cráneo que Prescott tanto ansiaba tocar era la prueba material, su prueba, para reconstruir el horror que sintieron los españoles ante los tzompantlis (Wade, 2018). Para Prescott la barbarie de la otredad estaba inscrita en esos restos humanos.

El modo en que una cultura define o cataloga un objeto está firmemente arraigado en el tiempo y el espacio. Las calaveras pertenecen al pasado, pero Prescott las lee en el presente. Y ¿qué pasa cuando un objeto viaja a través del tiempo y entre culturas? Aquí es donde la narración se vuelve clave. Como enfatiza Roland Barthes (1990), en el mundo objeto los objetos no tienen esencia, se refugian en sus atributos. Al despojar la calavera de su contexto, Prescott le asigna otro significado a través de su narración. No sabemos nada de sus atributos, ni de su forma o tamaño; esa pérdida es sustituida por la historia que la contiene y la narración que la define. En Historia de la conquista de México, la voz mexicana queda en el pasado, pero el objeto, representante simbólico de esa voz, queda en manos de Prescott quien le otorga un significado presente, corroborado por la ciencia. El pedido de Prescott refleja su deseo y su necesidad de materializar la cultura azteca en el presente. Como si en el cráneo mismo se encerrara el secreto del pasado indígena -es la posibilidad de hacer presente la barbarie-, como el niño en los escritos de Walter Benjamin quien, al tocar un juguete antiguo, puede recuperar la historia de su creación (Benjamin, 1986: 123).

La historia de los objetos y sus transformaciones demuestra cómo los objetos se vuelven inseparables del discurso y la cultura que los nombra. Para Prescott la barbarie de la otredad estaba inscrita en esos restos humanos. Prescott no tiene reparos en

Warren fue uno de los fundadores del mayor hospital de Boston, el Massachusetts General Hospital. El Warren Anatomical Museum hoy es parte de la biblioteca Countway de la escuela de medicina de Harvard University y contiene más de quince mil piezas.

24 Los otomíes fueron uno de los pueblos originarios que habitaron las áreas centrales de México, avasallados primero por grupos nahuas y luego por los mexica antes de ser conquistados por los españoles. Sus descendientes habitan hoy áreas del altiplano nor-oriente central de México (agradezco a Eulogio Guzmán por la aclaración).

25 "Recibidos dos cráneos mexicanos, aztecas, de W.H. Prescott quien, sin habérselo pedido se tomó el trabajo de hacerlos enviar desde el valle de México”. Lamentablemente, los cráneos hoy no se encuentran en la colección. 
querer conseguir una calavera, ni piensa que su afán lo acerque a las razas salvajes, porque como explica Fabian, la ciencia había creado una excusa (alibi) que legitimaba la colección de cráneos (Fabian, 2010: 12). La ciencia en esta instancia funciona como un eje paradigmático que, por un lado, determina cómo observar, catalogar y definir el valor de ciertos objetos y por otro, permite encubrir la inmanencia y simbología que se les otorgan a las cosas. Al recibir la carta confirmando el hallazgo de las calaveras, Prescott le escribió a Fanny Calderón: "Do not vex yourself any more about the skulls. The Otomite will answer all my wants" (Prescott en Wolcott, 230). Otomí o azteca, la diferencia poco importaba, la calavera era prueba suficiente de la barbarie, era lo único que Prescott necesitaba. 


\section{Q Bibliografía}

" Adorno, T. (1962). Prismas: La crítica de la cultura y la sociedad. Barcelona: Ariel.

" Appadurai, A. (1991). “Introducción: Las mercancías y la política de valor”. En: Appadurai, A. (ed.), La vida social de las cosas: Perspectiva cultural de las mercancías. México: Grijalbo, 17-88.

» Barthes, R. (1990). “Semántica del objeto”. Revista de Occidente, 104, 5-18.

"Benjamin, W. (1986). "Russian Toys". Moscow Diary. Cambridge: Harvard University Press, 123-124, trad. Gary Smith.

"Brown, B. (2001). “Thing Theory”. Critical Inquiry 28.1. En: http://www.jstor. com/stable/1344258; obtenido 13/07/2020.

" Cockcroft, J. D. (1968). "Prescott and His Sources: A Critical Appraisal”. The Hispanic American Historical Review, 48.1, 59-74.

"Cortés, E. y L. Gómez (2015). "Hispanismo y hegemonía en las Américas". Revista de Crítica Literaria Latinoamericana, 41-82.

"Dolan, J.R. (1964). The Yankee Peddlars of Early America. New York: Clarkson N. Potter.

" Eipper, J. (2000). “The Canonizer De-Canonized: The Case of William H. Prescott”. Hispania, 83.3, 416-427.

»Ette, O. (2019). Alexander von Humboldt y la globalización: el saber en movimiento. México: El Colegio de México. Trad. Johanna Malcher.

»Fabian, A. (2010). The Skull Collectors: Race, Science, and America's Unburied Dead. Chicago: The University of Chicago Press.

» Filler, L. \& A. Guttmann (1962). The Removal of the Cherokee Nation: Manifest Destiny or National Dishonor. Boston: Heath.

»Franey, L. (2001). “Ethnographic Collecting and Travel: Blurring Boundaries, Forming a Discipline". Victorian Literature and Culture, 219-239.

" Gerassi-Navarro, N. (2017). Women, Travel and Science in Nineteenth-Century Americas: The Politics of Observation. New York: Palgrave Macmillan.

»Gómez, L. (2015). “El hispanismo en viaje: William H. Prescott y México”. Revista de Crítica Literaria Latinoamericana, 61.82, 117-134.

» Gould, S.J. (1996). The Mismeasure of Man. New York: Norton \& Company.

» Grassby, R. (2005). “Material Culture and Cultural History”. The Journal of Interdisciplinary History, 35.4, 591-603.

» Hick, D. y M. Beaudry (2010). Material Culture Studies: A Reactionary View. The Oxford Handbook of Material Culture. Oxford: Oxford University Press, 1-21.

" Jaksic, I. (2007). The Hispanic World and the American Intellectual Life. New York: Palgrave Macmillan.

" Jaffee, D. (1991). "Peddlers of Progress and the Transformation of the Rural North, 1760-1860". The Journal of American History, 18.2, 511-535.

" Jaramillo Uribe, J. (1986). "Frecuencias temáticas de la historiografía latinoamericana”. En: Zea, L. (coord.), América Latina en sus ideas. México, UNESCO: 
Siglo XXI, 23-45.

"Johannsen, R. W. (1985). To the Halls of the Montezumas: the Mexican War in the American Imagination. New York: Oxford University Press.

» Kagan, R. (1996). “Prescott's Paradigm: American Historical Scholarship and The Decline of Spain". The American Historical Review, 101.2, 423-446.

"Keen, B. (1990). The Aztec Image in Western Thought. New Brunswick: Rutgers University Press.

»Koch, P.O. (2016). William Hickling Prescott: The Life and Letters of America's First Scientific Historian. Jefferson, North Carolina: MacFarland and Company.

» Lagunas Rodríguez, Z. (2004). “El uso ritual del cuerpo en el México prehispánico". Arqueología mexicana, XI, 42-47.

"Latour, B. (2000). “The Berlin Key or How To Do Words with Things". Matter, Materiality, and Modern Culture. En: http://www.bruno-latour.fr/sites/default/ files/P-36-Berliner-KEY-GBpdf.pdf; obtenido el 14/07/2020.

»Levin, D. (1959). History as Romantic Art. Stanford: Stanford University Press.

» Lipset, S. M. (1996). American Exceptionalism: A Double-Edged Sword. New York: Norton.

»Lukács, G. (1971). La novela histórica. México: Era.

» Moraña, M. (2005). Ideologies of Hispanism. Nashville: Vanderbilt University Press.

"Morton, S. (1839). Crania Americana or a Comparative View of the Skulls of Various Aboriginal Nations of North and South America. Philadelphia: Dobson.

"O'Sullivan, J. L. (1839). “The Great Nation of Futurity”. The United States Democratic Review, 6.23, 426-430.

»O'Sullivan, J. L. (1845). “Annexation”. The United States Magazine and Democratic Review, 17. En: https://pdcrodas.webs.ull.es/anglo/OSullivanAnnexation.pdf; obtenido 17/07/2020.

»Peck, H. T. (1926). William Hickling Prescott. New York: The Macmillan Company.

» Prescott, W. H. (2001) [1843]. History of the Conquest of Mexico. New York: Modern Library.

»Prescott, W. H. (1961). The Literary Memoranda of William Hickling Prescott 2 v. ed. C. Harvey

» Gardiner. Norman: University of Oklahoma Press.

»Rouvière, H. y A. Delmas, (2006). Anatomía humana, descriptiva, topográfica y funcional vol.1 Barcelona: Masson.

»Talavera González, J. A. (2008). “EL aprovechamiento del cuerpo humano en el México prehispánico”. Arqueología Mexicana, 16.91, 69-70.

"Tenenbaum, B. (1979). "Merchants, Money, and Mischief: The British in Mexico, 1821-1862”. The Americas, 35.3, 317-339.

» Ticknor, G. (1865). The Life of William Hickling Prescott. Philadelphia: J. B. Lippincott Co.

"Torres Monroy, L. (2000). "Naturaleza de las colecciones documentales: el problema de su tratamiento archivístico". En: Villanueva Bazán, G. (coord.), Teoría y práctica archivística 1. México: Universidad Autónoma de México, 31-40. 
»Ulrich, L.T., I. Gaskell, S. J. Schechner, S. A. Carter with photographs by S. S.B. Van Gerbig. (2015). Tangible Things: Making History through Objects. Oxford: Oxford University Press.

»Wade, L. (2018). "Feeding the Gods: Hundreds of Skulls Reveal Massive Scale of Human Sacrifice in Aztec Capital”.

»En: https://www.sciencemag.org/news/2018/o6/feeding-gods-hundreds-skullsreveal-massive-scale-human-sacrifice-aztec-capital; obtenido el 26/07/2020.

»Wade, N. (2011). "Scientists Measure the Accuracy in a Racism Claim". The New York Times En: https://www.nytimes.com/2011/06/14/science/14skull. html?smid=em-share; obtenido el 21/07/2020.

»Warren, J. C. (1941). Papers, vol. 79, Ms N-1731.

"Weisberg, M y D. B. Paul (2016). “Morton, Gould, and Bias: A Comment on the “Mismeasure of Science”. En: https://doi.org/10.1371/journal.pbio.1002444; obtenido el 26/07/2020.

»Wolcott, R. Ed. (1925). The Correspondence of W. H. Prescott 1833-1847. Boston: Houghton Mifflin Company.

»Zuleta, E. de (1992). “El hispanismo de Hispanoamérica”. Hispania, 75.4, 995-965. https://journals.plos.org/plosbiology/article?id=10.1371/journal.pbio.1002444 
